\title{
Stability Analysis of Analytical and Numerical Solutions to Nonlinear Delay Differential Equations with Variable Impulses
}

\author{
X. Liu and Y. M. Zeng \\ School of Mathematical Sciences, Liaocheng University, Liaocheng 252059, China \\ Correspondence should be addressed to X. Liu; liuxing6213693@163.com
}

Received 17 April 2017; Revised 12 September 2017; Accepted 3 October 2017; Published 13 November 2017

Academic Editor: Mustafa R. S. Kulenovic

Copyright ( 2017 X. Liu and Y. M. Zeng. This is an open access article distributed under the Creative Commons Attribution License, which permits unrestricted use, distribution, and reproduction in any medium, provided the original work is properly cited.

A stability theory of nonlinear impulsive delay differential equations (IDDEs) is established. Existing algorithm may not converge when the impulses are variable. A convergent numerical scheme is established for nonlinear delay differential equations with variable impulses. Some stability conditions of analytical and numerical solutions to IDDEs are given by the properties of delay differential equations without impulsive perturbations.

\section{Introduction}

In this paper, we study the stability of nonlinear impulsive differential equations:

$$
\begin{aligned}
x^{\prime}(t) & =f(t, x(t), x(t-\tau)), \quad t \geq 0, t \neq k \tau, \\
\Delta x & =r_{k} x(t), \quad t=k \tau, \quad k=0,1, \ldots, \\
x(t) & =\varphi(t), \quad-\tau \leq t \leq 0,
\end{aligned}
$$

where $f:[0,+\infty) \times C^{q} \times C^{q} \rightarrow C^{q}, r_{k} \neq-1, \tau$ is a positive constant, $\varphi(t)$ is a continuous function on $[-\tau, 0)$, and $\lim _{t \rightarrow 0^{-}} \varphi(t)$ exists. Assume that $\Delta x=x(t+0)-x(t)$.

Definition 1 (see [1]). $x(t)$ is said to be the solution of (1), if

(1) $x(0)=\left(1+r_{0}\right) \lim _{t \rightarrow 0^{-}} \varphi(t)$,

(2) $x(t)$ is differentiable and $x^{\prime}(t)=f(t, x(t), x(t-\tau))$ for $t \in(0,+\infty), t>0, t \neq k \tau$,

(3) $x(t)$ is left continuous in $(0,+\infty)$ and $x\left(k \tau^{+}\right)=(1+$ $\left.r_{k}\right) x(k \tau), k=0,1,2, \ldots$

Impulsive differential equations are widely used in actual modeling such as epidemic, optimal control and population dynamics (see $[2,3]$ ). Up to now, extensive work has been done in the area of qualitative theories of IDDEs (see [4-6]). The numerical properties of impulsive differential equations attracted attentions of scholars since Ran et al's work in [7], which showed that the explicit Euler method is stable for impulsive differential equations, while the implicit Euler method is not. Some excellent works have been done in existing literature. In [8], the convergence of Euler method for linear IDDEs is studied. Zhang et al. studied the numerical stability of Runge-Kutta methods to IDDEs in $[9,10]$ and proved that backward Euler method and 2-stage Lobatto IIIC method can preserve the stability of nonlinear IDDEs with fixed impulses. Unfortunately, the numerical scheme may not converge when the impulses are variable. It is necessary to find a convergent stable method for IDDEs with variable pulses.

The rest of this paper is organized as follows. In Section 2, we establish a theorem that enables us to transform the stability of IDDEs to corresponding delay differential equations without impulsive perturbations. In Sections 3 and 4, stability of exact solutions is studied. In Section 5, a convergent numerical scheme is constructed. In Section 6, the stability of numerical solutions is researched. In Section 7, numerical experiments are given to confirm the conclusions.

\section{Preliminaries}

Recently, a new technique has been introduced in the stability analysis of exact and numerical solutions to impulsive differential equations by constructing equivalent equations 
(see [9-12]). But the equivalent equations may not work when the impulses are variable. In this section, we will propose an improved equivalent equation.

Hypothesis 1. Assume that the function $\alpha(t)$ satisfies the following:

(1) $\alpha(t)$ is continuously differentiable on $(k \tau,(k+1) \tau)$, $k=0,1, \ldots$.

(2) $\alpha\left(k \tau^{-}\right)=\left(1+r_{k}\right) \alpha\left(k \tau^{+}\right), k=0,1, \ldots$

(3) $\alpha(t) \neq 0, \forall t>0$.

Remark 2. It is easy to check that

$$
\begin{aligned}
& \alpha(t)=\left(1+r_{k}\right)^{(t-(k+1) \tau) / \tau}, \quad t \in(k \tau,(k+1) \tau], \\
& \alpha(t)=\prod_{i=1}^{k} \frac{1}{1+r_{k}}, \quad t \in(k \tau,(k+1) \tau]
\end{aligned}
$$

satisfy Hypothesis 1.

Consider the equation

$$
\begin{aligned}
& y^{\prime}(t)=\frac{\alpha^{\prime}(t)}{\alpha(t)} y(t)+\alpha(t) f\left(t, \frac{y(t)}{\alpha(t)}, \frac{y(t-\tau)}{\alpha(t-\tau)}\right), \\
& y(t)=\varphi(t), \quad-\tau \leq t \leq 0,
\end{aligned}
$$

where $\alpha(t)$ has been defined in Hypothesis 1 . Here $\alpha^{\prime}(t)$ is the left derivative of $\alpha(t)$.

Corollary 3. In the case that $r_{k}=r, k=0,1,2, \ldots$, the transformation function (2) can be reduced to $\alpha(t)=(1 /(1+$ $r))^{\{t / \tau\}-1}$. Then (4) is the same as the equivalent equations proposed in $[9,13]$.

Theorem 4. Assume that Hypothesis 1 holds.

(1) If $x(t)$ is the solution of (1), then $y(t)=\alpha(t) x(t)$ is the solution of (4).

(2) If $y(t)$ is the solution of (4), then $x(t)=y(t) / \alpha(t)$ is the solution of (1).

Proof. (1) It is obvious that $y(t)$ is continuous on each interval $(k \tau,(k+1) \tau)$. On the other hand, for any $k=0,1,2, \ldots$,

$$
\begin{aligned}
y\left(k \tau^{+}\right) & =x\left(k \tau^{+}\right) \alpha\left(k \tau^{+}\right)=\left(1+r_{k}\right) x\left(k \tau^{-}\right) \alpha\left(k \tau^{+}\right) \\
& =\alpha\left(k \tau^{-}\right) x\left(k \tau^{-}\right)=y\left(k \tau^{-}\right),
\end{aligned}
$$

which implies that $y(t)$ is continuous in $[0,+\infty)$. It is easy to verify that $y(t)=\alpha(t) x(t)$ is the solution of (4).

(2) On each interval $(k \tau,(k+1) \tau)$,

$$
\begin{aligned}
& x^{\prime}(t)=\frac{y^{\prime}(t) \alpha(t)-y(t) \alpha^{\prime}(t)}{\alpha^{2}(t)} \\
& =\frac{\left(\alpha^{\prime}(t) / \alpha(t)\right) y(t)+\alpha(t) f(t, y(t) / \alpha(t), y(t-\tau) / \alpha(t-\tau))}{\alpha(t)} \\
& -\frac{y(t) \alpha^{\prime}(t)}{\alpha^{2}(t)}=f\left(t, \frac{y(t)}{\alpha(t)}, \frac{y(t-\tau)}{\alpha(t-\tau)}\right)=f(t, x(t), x(t-\tau)) .
\end{aligned}
$$

On the other hand, at each impulsive point $t=k \tau$,

$$
\begin{aligned}
x\left(k \tau^{+}\right) & =\lim _{t \rightarrow k \tau^{+}} \frac{y(t)}{\alpha(t)}=\frac{y\left(k \tau^{+}\right)}{\alpha\left(k \tau^{+}\right)}=\frac{\left(1+r_{k}\right) y\left(k \tau^{-}\right)}{\alpha\left(k \tau^{-}\right)} \\
& =\left(1+r_{k}\right) x\left(k \tau^{-}\right) .
\end{aligned}
$$

It follows from Definition 1 that $x(t)$ is the solution of (1).

\section{Stability Analysis of Exact Solutions}

Let $\langle\cdot, \cdot\rangle$ be an inner product on $C^{q}$ and $\|\cdot\|$ is the corresponding norm. We assume that there exist real constants $\alpha$ and $\beta$ such that the function $f$ in (1) satisfies

$$
\begin{array}{r}
\Re\left\langle x_{1}-x_{2}, f\left(t, x_{1}, y\right)-f\left(t, x_{2}, y\right)\right\rangle \leq \alpha\left\|x_{1}-x_{2}\right\|^{2}, \\
\left\|f\left(t, x, y_{1}\right)-f\left(t, x, y_{2}\right)\right\| \leq \beta\left\|y_{1}-y_{2}\right\| .
\end{array}
$$

To study the stability of (1), we also consider the equation with differential initial value:

$$
\begin{aligned}
\bar{x}^{\prime}(t) & =f(t, \bar{x}(t), \bar{x}(t-\tau)), \quad t \geq 0, t \neq k \tau, \\
\Delta \bar{x} & =r_{k} \bar{x}(t), \quad t=k \tau, k=0,1, \ldots, \\
\bar{x}(t) & =\psi(t), \quad-\tau \leq t \leq 0 .
\end{aligned}
$$

Based on the transformation

$$
z(t)=\alpha(t) \bar{x}(t),
$$

we can get an equivalent equation to (9) as follows:

$$
\begin{aligned}
& z^{\prime}(t)=\frac{\alpha^{\prime}(t)}{\alpha(t)} z(t)+\alpha(t) f\left(t, \frac{z(t)}{\alpha(t)}, \frac{z(t-\tau)}{\alpha(t-\tau)}\right), \\
& \quad t>0, \\
& z(t)=\psi(t), \quad-\tau \leq t \leq 0 .
\end{aligned}
$$

Definition 5. The zero solution of (1) is said to be stable, if there exists a constant $C$ such that

$$
\|x(t)-\bar{x}(t)\| \leq C \sup _{-\tau \leq s \leq 0}\|\varphi(s)-\psi(s)\| .
$$

Definition 6. The zero solution of (1) is said to be asymptotically stable, if

$$
\lim _{t \rightarrow+\infty}\|x(t)-\bar{x}(t)\|=0 .
$$

Lemma 7. Assume that

$$
\begin{gathered}
\Re\left(\frac{\alpha^{\prime}(t)}{\alpha(t)}\right)+\alpha \leq \alpha_{0}, \\
\left|\beta \frac{\alpha(t)}{\alpha(t-\tau)}\right| \leq \beta_{0}
\end{gathered}
$$

hold. Then

(1) if $\alpha_{0}+\beta_{0} \leq 0$,

$$
\|y(t)-z(t)\| \leq \sup _{-\tau \leq s \leq 0}\|\varphi(s)-\psi(s)\|,
$$


(2) if $\alpha_{0}+\beta_{0}<0$,

$$
\lim _{t \rightarrow+\infty}\|y(t)-z(t)\|=0 .
$$

Proof. Note that

$$
\begin{aligned}
& \frac{1}{2} \frac{d}{d t}\|y(t)-z(t)\|^{2}=\Re\left\langle y(t)-z(t), \frac{\alpha^{\prime}(t)}{\alpha(t)}(y(t)\right. \\
& -z(t))+\alpha(t)\left[f\left(t, \frac{y(t)}{\alpha(t)}, \frac{y(t-\tau)}{\alpha(t-\tau)}\right)\right. \\
& \left.\left.-f\left(t, \frac{z(t)}{\alpha(t)}, \frac{z(t-\tau)}{\alpha(t-\tau)}\right)\right]\right\rangle=\Re \frac{\alpha^{\prime}(t)}{\alpha(t)} \| y(t) \\
& -z(t) \|^{2}+\Re\langle y(t)-z(t), \alpha(t) \\
& \cdot\left[f\left(t, \frac{y(t)}{\alpha(t)}, \frac{y(t-\tau)}{\alpha(t-\tau)}\right)\right. \\
& \left.\left.-f\left(t, \frac{z(t)}{\alpha(t)}, \frac{y(t-\tau)}{\alpha(t-\tau)}\right)\right]\right\rangle+\Re\langle y(t)-z(t), \\
& \alpha(t)\left[f\left(t, \frac{z(t)}{\alpha(t)}, \frac{y(t-\tau)}{\alpha(t-\tau)}\right)\right. \\
& \left.\left.-f\left(t, \frac{z(t)}{\alpha(t)}, \frac{z(t-\tau)}{\alpha(t-\tau)}\right)\right]\right\rangle \leq \Re\left(\frac{\alpha^{\prime}(t)}{\alpha(t)}+\alpha\right) \\
& \cdot\|y(t)-z(t)\|^{2}+\beta\left|\frac{\alpha(t)}{\alpha(t-\tau)}\right| \cdot\|y(t)-z(t)\| \\
& \cdot\|y(t-\tau)-z(t-\tau)\| \leq \alpha_{0}\|y(t)-z(t)\|^{2}+\beta_{0} \\
& \cdot\|y(t)-z(t)\| \cdot\|y(t-\tau)-z(t-\tau)\| .
\end{aligned}
$$

Therefore,

$$
\begin{aligned}
\frac{d}{d t}\|y(t)-z(t)\| \leq & \alpha_{0}\|y(t)-z(t)\| \\
& +\beta_{0}\|y(t-\tau)-z(t-\tau)\| .
\end{aligned}
$$

It follows that

$$
\begin{aligned}
& \|y(t)-z(t)\| \\
& \leq\left[e^{\alpha_{0} \tau}+\frac{\beta_{0}}{\alpha_{0}}\left(e^{\alpha_{0} \tau}-1\right)\right]^{k} \sup _{-\tau \leq s \leq 0}\|\varphi(s)-\psi(s)\|, \\
& \quad t \in(k \tau,(k+1) \tau] .
\end{aligned}
$$

On the other hand, $e^{\alpha_{0} \tau}+\left(\beta_{0} / \alpha_{0}\right)\left(e^{\alpha_{0} \tau}-1\right) \in(0,1]$ when $\alpha_{0}+\beta_{0} \leq 0$ and $\left|e^{\alpha_{0} \tau}+\left(\beta_{0} / \alpha_{0}\right)\left(e^{\alpha_{0} \tau}-1\right)\right|<1$ when $\alpha_{0}+\beta_{0}<0$. The proof is complete. of (1).

By Theorem 4 and Lemma 7, we can obtain the stability

Theorem 8. Assume that $1 / \alpha(t)$ is bounded. Then

(1) if $\alpha_{0}+\beta_{0} \leq 0$, then zero solution of (1) is stable,
(2) if $\alpha_{0}+\beta_{0}<0$, then zero solution of (1) is asymptotically stable.

To investigate the stability of (1) by Theorem 8 , we need to establish a suitable function $\alpha(t)$. Different stability conditions may be given under different function $\alpha(t)$. In the next section, some specific stability conditions are given.

\section{Some Specific Stability Conditions}

In this section, some specific stability conditions are given based on the choice of $\alpha(t)$ proposed in (2) and (3).

Case 1 (take $\alpha(t)$ as in (2)). Taking $\alpha(t)$ as in (2), we can obtain the following conclusions by Theorem 8 .

Theorem 9. Assume that

$$
\begin{gathered}
\left|\beta\left(\frac{1+r_{k}}{1+r_{k-1}}\right)^{(t-(k+1) \tau) / \tau}\right| \leq \beta_{0}, \quad t \in(k \tau,(k+1) \tau], \\
\frac{\ln \left(1+r_{k}\right)}{\tau}+\alpha \leq \alpha_{0}, \quad k=0,1,2, \ldots
\end{gathered}
$$

Then the zero solution of (1) is asymptotically stable if $\beta_{0}<-\alpha_{0}$.

Remark 10. The zero solution of (1) is asymptotically stable if $\beta<-(\ln (1+r) / \tau+\alpha)$, when $r_{k}=r, k=0,1, \ldots$. The zero solution of (1) is asymptotically stable if $\beta<-\alpha$, when $r_{k}=0$, $k=0,1, \ldots$. Therefore, in the case that impulses are fixed or absent, the conclusions in Theorem 8 can be reduced to the same as in $[9,14]$.

Corollary 11. Assume that

$$
1 \geq\left|1+r_{k}\right| \geq\left|1+r_{k-1}\right|, \quad k=0,1,2, \ldots
$$

Then the zero solution of (1) is asymptotically stable if $\beta<-\alpha$.

Case 2 (take $\alpha(t)$ as in (3)). Taking $\alpha(t)$ as in (3), we can obtain the following conclusion by Theorem 8 .

Corollary 12. If there exists a constant $M$, the following hold:

$$
\begin{gathered}
\left|\prod_{i=1}^{k}\left(1+r_{i}\right)\right| \leq M, \quad t \in(k \tau,(k+1) \tau], \\
\left|\frac{\beta}{1+r_{k}}\right| \leq \beta_{0}, \quad k=0,1,2, \ldots
\end{gathered}
$$

The zero solution of (1) is stable when $\beta_{0} \leq-\alpha_{0}$ and is asymptotically stable when $\beta_{0}<-\alpha_{0}$.

Note that it is difficult to find sequences $\left\{r_{k}\right\}$ satisfying conditions (22) and (23) simultaneously. It is necessary to find other stability conditions in this choice of $\alpha(t)$. 
Theorem 13. Assume that the following holds:

$$
\begin{aligned}
\left|1+r_{i}\right| & \leq 1, \quad i=1,2, \ldots, \\
\frac{1}{\alpha(t)} & \leq \prod_{i=1}^{k}\left|1+r_{i}\right|, \quad t \in(k \tau,(k+1) \tau] .
\end{aligned}
$$

Then the zero solution of (1) is stable when $\beta \leq-\alpha$ and is asymptotically stable when $\beta<-\alpha$.

Proof. Note that while we take $\alpha(t)$ as in (3), inequality (17) becomes

$$
\begin{array}{rl}
\frac{d}{d t}\|y(t)-z(t)\| \leq & \alpha\|y(t)-z(t)\| \\
& +\frac{\beta}{\left|1+r_{k}\right|}\|y(t-\tau)-z(t-\tau)\|, \\
t & t \in(k \tau,(k+1) \tau] .
\end{array}
$$

Hence,

$$
\begin{aligned}
\|y(t)-z(t)\| \leq & \prod_{i=1}^{k}\left[e^{\alpha \tau}+\frac{\beta}{\left|1+r_{i}\right| \alpha}\left(e^{\alpha \tau}-1\right)\right] \\
& \cdot \sup _{-\tau \leq s \leq 0}\|\varphi(s)-\psi(s)\| .
\end{aligned}
$$

By Theorem 4 and inequality (17), for $t \in(k \tau,(k+1) \tau]$, we have

$$
\begin{aligned}
& \|x(t)-\bar{x}(t)\|=\left\|\frac{y(t)-z(t)}{\alpha(t)}\right\| \leq\left|\frac{1}{\alpha(t)}\right| \\
& \cdot \prod_{i=1}^{k}\left[e^{\alpha \tau}+\frac{\beta}{\left|1+r_{i}\right| \alpha}\left(e^{\alpha \tau}-1\right)\right] \\
& \cdot \sup _{-\tau \leq s \leq 0}\|\varphi(s)-\psi(s)\| \leq\left[e^{\alpha \tau}+\frac{\beta}{\alpha}\left(e^{\alpha \tau}-1\right)\right]^{k} \\
& . \sup _{-\tau \leq s \leq 0}\|\varphi(s)-\psi(s)\| .
\end{aligned}
$$

Note that $0<e^{\alpha \tau}+(\beta / \alpha)\left(e^{\alpha \tau}-1\right) \leq 1$ if $\beta \leq-\alpha$ and $0<$ $e^{\alpha \tau}+(\beta / \alpha)\left(e^{\alpha \tau}-1\right)<1$ if $\beta<-\alpha$. The proof is complete.

\section{Numerical Process}

In this section, we establish a convergent numerical process of $\theta$ methods for (1).

5.1. $\theta$ Methods for Ordinary Differential Equations. The application of one-stage $\theta$ method in case of an ordinary differential equation,

$$
y^{\prime}(t)=f(t, y(t))
$$

yields

$$
\begin{aligned}
Y_{n} & =y_{n}+h \theta f\left(t_{n}+\theta h, Y_{n}\right), \\
y_{n+1} & =y_{n}+h f\left(t_{n}+\theta h, Y_{n}\right),
\end{aligned}
$$

where $h$ denotes the step size and $Y_{n}$ is an approximation of $y\left(t_{n}+\theta h\right)$.
5.2. $\theta$ Methods for IDDEs. Let $h=\tau / m$ be a given step size with integer $m \geq 1$. The application of one-stage $\theta$ methods, in case of $(4)$ on interval $(k \tau,(k+1) \tau]$, yields

$$
\begin{gathered}
y_{n+1}=y_{n}+h\left[\frac{\alpha^{\prime}\left(t_{n}+\theta h\right)}{\alpha\left(t_{n}+\theta h\right)} Y_{n}+\alpha\left(t_{n}+\theta h\right)\right. \\
\left.\cdot f\left(t_{n}+\theta h, \frac{Y_{n}}{\alpha\left(t_{n}+\theta h\right)}, \frac{Y_{n-m}}{\alpha\left(t_{n}+\theta h-\tau\right)}\right)\right], \\
Y_{n}=y_{n}+h \theta\left[\frac{\alpha^{\prime}\left(t_{n}+\theta h\right)}{\alpha\left(t_{n}+\theta h\right)} Y_{n}+\alpha\left(t_{n}+\theta h\right)\right. \\
\left.\cdot f\left(t_{n}+\theta h, \frac{Y_{n}}{\alpha\left(t_{n}+\theta h\right)}, \frac{Y_{n-m}}{\alpha\left(t_{n}+\theta h-\tau\right)}\right)\right],
\end{gathered}
$$

where $y_{n}$ denotes an approximation to $y\left(t_{n}\right)$ for $n>0$. Based on Theorem 4, the solution of (1) can be approximated by (30) and

$$
x_{n+1}=\frac{y_{n+1}}{\alpha\left(t_{n+1}\right)} \text {. }
$$

In the same way, we can define the numerical solutions of (3).

$$
\begin{aligned}
& z_{n+1}=z_{n}+h\left[\frac{\alpha^{\prime}\left(t_{n}+\theta h\right)}{\alpha\left(t_{n}+\theta h\right)} Z_{n}+\alpha\left(t_{n}+\theta h\right)\right. \\
& \left.\cdot f\left(t_{n}+\theta h, \frac{Z_{n}}{\alpha\left(t_{n}+\theta h\right)}, \frac{Z_{n-m}}{\alpha\left(t_{n}+\theta h-\tau\right)}\right)\right], \\
& Z_{n}=z_{n}+h \theta\left[\frac{\alpha^{\prime}\left(t_{n}+\theta h\right)}{\alpha\left(t_{n}+\theta h\right)} Z_{n}+\alpha\left(t_{n}+\theta h\right)\right. \\
& \left.\cdot f\left(t_{n}+\theta h, \frac{Z_{n}}{\alpha\left(t_{n}+\theta h\right)}, \frac{Z_{n-m}}{\alpha\left(t_{n}+\theta h-\tau\right)}\right)\right], \\
& \bar{x}_{n+1}=\frac{z_{n+1}}{\alpha\left(t_{n+1}\right)} .
\end{aligned}
$$

Remark 14. If we take $\alpha(t)$ as in (2), numerical processes (30) and (31) can be reduced to

$$
\begin{gathered}
y_{n+1}=y_{n}+h\left[\frac{\ln \left(1+r_{k}\right)}{\tau} Y_{n}+\alpha\left(t_{n}+\theta h\right)\right. \\
\left.\cdot f\left(t_{n}+\theta h, \frac{Y_{n}}{\alpha\left(t_{n}+\theta h\right)}, \frac{Y_{n-m}}{\alpha\left(t_{n}+\theta h-\tau\right)}\right)\right], \\
Y_{n}=y_{n}+h \theta\left[\frac{\ln \left(1+r_{k}\right)}{\tau} Y_{n}+\alpha\left(t_{n}+\theta h\right)\right. \\
\left.\cdot f\left(t_{n}+\theta h, \frac{Y_{n}}{\alpha\left(t_{n}+\theta h\right)}, \frac{Y_{n-m}}{\alpha\left(t_{n}+\theta h-\tau\right)}\right)\right],
\end{gathered}
$$

when $t_{n} \in(k \tau,(k+1) \tau]$. 
Remark 15. If we take $\alpha(t)$ as in (3), numerical processes (30) and (31) can be reduced to

$$
\begin{aligned}
y_{n+1} & =y_{n}+h \alpha\left(t_{n}+\theta h\right) \\
\cdot & f\left(t_{n}+\theta h, \frac{Y_{n}}{\alpha\left(t_{n}+\theta h\right)}, \frac{Y_{n-m}}{\alpha\left(t_{n}+\theta h-\tau\right)}\right), \\
Y_{n}= & y_{n}+h \theta \alpha\left(t_{n}+\theta h\right) \\
& \cdot f\left(t_{n}+\theta h, \frac{Y_{n}}{\alpha\left(t_{n}+\theta h\right)}, \frac{Y_{n-m}}{\alpha\left(t_{n}+\theta h-\tau\right)}\right) .
\end{aligned}
$$

The convergence of numerical processes (30) and (31) is described in the following theorem.

Theorem 16. Assume that $\alpha(t)$ is continuously differentiable on $(k \tau,(k+1) \tau), k=0,1,2, \ldots$, and $1 / \alpha(t)$ is bounded. Then numerical processes (30) and (31) are of order 1 when $\theta \neq 1 / 2$ and of order 2 when $\theta=1 / 2$.

Proof. On each interval $(k \tau,(k+1) \tau]$, (4) reduces to a DDE. Therefore $y_{n}-y\left(t_{n}\right)=O(h)$ for $\theta \neq 1 / 2$ and $y_{n}-y\left(t_{n}\right)=O\left(h^{2}\right)$ for $\theta=1 / 2$. On the other hand, $\left|x_{n}-x\left(t_{n}\right)\right|=\mid\left(1 / \alpha\left(t_{n}\right)\right)\left(y_{n}-\right.$ $\left.y\left(t_{n}\right)\right) \mid$. The conclusion follows because $\alpha(t)$ is bounded.

\section{Stability Analysis of Numerical Solutions}

In this section, we study the stability property of numerical solutions. As usual, we expect that the numerical solution can reproduce the property of the exact solutions. The stability of the numerical solutions can be defined according to Theorem 4.

Definition 17. A numerical method is said to be stable for (1), if there exists a constant $C$, such that the numerical solutions $x_{n}$ and $\bar{x}_{n}$ of (1) and (4) satisfy

$$
\left\|x_{n}-\bar{x}_{n}\right\| \leq C \cdot \max _{-\tau \leq s \leq 0}\|\varphi(s)-\psi(s)\|
$$

for every step size $h$ under the constraint $h m=\tau$, where $m$ is a positive integer.

Definition 18. A numerical method is said to be asymptotically stable for (1), if the numerical solutions $x_{n}$ and $\bar{x}_{n}$ of (1) and (4) satisfy (35) and

$$
\lim _{n \rightarrow+\infty}\left\|x_{n}-\bar{x}_{n}\right\|=0
$$

for every step size $h$ under the constraint $h m=\tau$, where $m$ is a positive integer.
Denote

$$
\begin{aligned}
\widehat{f}(t, y, z)= & \frac{\alpha^{\prime}(t)}{\alpha(t)} y+\alpha(t) f\left(t, \frac{y}{\alpha(t)}, \frac{z}{\alpha(t-\tau)}\right), \\
\delta_{n}= & y_{n}-z_{n}, \\
\omega_{n}= & Y_{n}-Z_{n}, \\
K_{n}= & \widehat{f}\left(t_{n}+\theta h, Y_{n}, Y_{n-m}\right) \\
& -\widehat{f}\left(t_{n}+\theta h, Z_{n}, Y_{n-m}\right), \\
H_{n}= & \widehat{f}\left(t_{n}+\theta h, Z_{n}, Y_{n-m}\right) \\
& -\widehat{f}\left(t_{n}+\theta h, Z_{n}, Z_{n-m}\right), \\
\Delta_{n}= & \widehat{f}\left(t_{n}+\theta h, Y_{n}, Y_{n-m}\right) \\
& -\widehat{f}\left(t_{n}+\theta h, Z_{n}, Y_{n-m}\right) .
\end{aligned}
$$

It follows from (30) that

$$
\begin{gathered}
\omega_{n}=\delta_{n}+h \theta \Delta_{n}, \\
\delta_{n+1}=\delta_{n}+h \Delta_{n} .
\end{gathered}
$$

Lemma 19. Assume that (8) holds. Then

$$
\left\|\delta_{n+1}\right\|^{2} \leq\left\|\delta_{n}\right\|^{2}+h\left(2 \alpha_{0}+\beta_{0}\right)\left\|\omega_{n}\right\|^{2}+h \beta_{0}\left\|\omega_{n-m}\right\|^{2}
$$

holds if $\theta \geq 1 / 2$. Furthermore, if $\alpha_{0}+\beta_{0} \leq 0$, inequality

$$
\left\|\delta_{n+1}\right\|^{2} \leq\left\|\delta_{n}\right\|^{2}+h \alpha_{0}\left\|\omega_{n}\right\|^{2}+h \beta_{0}\left\|\omega_{n-m}\right\|^{2}
$$

holds.

Proof. By difference equation (38), it is obvious that

$$
\begin{aligned}
& \left\|\delta_{n+1}\right\|^{2}=\left\|\delta_{n}+h \Delta_{n}\right\|^{2}=\left\|\delta_{n}\right\|^{2}+2 h \Re\left\langle\omega_{n}, \Delta_{n}\right\rangle-(2 \theta \\
& -1) h^{2} \Re\left\langle\Delta_{n}, \Delta_{n}\right\rangle \leq\left\|\delta_{n}\right\|^{2}+2 h \Re\left\langle\omega_{n}, K_{n}+H_{n}\right\rangle \\
& =\left\|\delta_{n}\right\|^{2}+2 h \Re\left\langle\omega_{n}, K_{n}\right\rangle+2 h \Re\left\langle\omega_{n}, H_{n}\right\rangle \leq\left\|\delta_{n}\right\|^{2} \\
& +2 h\left[\Re \frac{\alpha^{\prime}\left(t_{n}+\theta h\right)}{\alpha\left(t_{n}+\theta h\right)}+\alpha\right]\left\|\omega_{n}\right\|^{2} \\
& +2 h \beta\left|\frac{\alpha\left(t_{n}+\theta h\right)}{\alpha\left(t_{n}+\theta h-\tau\right)}\right| \cdot\left\|\omega_{n}\right\| \cdot\left\|\omega_{n-m}\right\| \leq\left\|\delta_{n}\right\|^{2} \\
& +\left[2 h\left(\Re \frac{\alpha^{\prime}\left(t_{n}+\theta h\right)}{\alpha\left(t_{n}+\theta h\right)}+\alpha\right)\right. \\
& \left.+h \beta\left|\frac{\alpha\left(t_{n}+\theta h\right)}{\alpha\left(t_{n}+\theta h-\tau\right)}\right|\right]\left\|\omega_{n}\right\|^{2} \\
& +h \beta\left|\frac{\alpha\left(t_{n}+\theta h\right)}{\alpha\left(t_{n}+\theta h-\tau\right)}\right| \cdot\left\|\omega_{n-m}\right\|^{2} \leq\left\|\delta_{n}\right\|^{2}+h\left(2 \alpha_{0}\right. \\
& \left.+\beta_{0}\right)\left\|\omega_{n}\right\|^{2}+h \beta_{0}\left\|\omega_{n-m}\right\|^{2} .
\end{aligned}
$$

The proof is complete. 
Using a similar technique to the one in $[15,16]$, we can obtain the numerical stability of $\theta$ methods to (1).

Theorem 20. Assume that $1 / \alpha(t)$ is bounded and $\theta \in[1 / 2,1]$. Then the numerical methods (30) and (31) are asymptotically stable for (1) with

$$
\beta_{0}<\frac{2(2 \theta-1)^{2}}{(2 \theta-1)^{2}+1}\left(-\alpha_{0}\right) .
$$

Proof. It follows from (38) that

$$
w_{n}=\theta \delta_{n+1}+(1-\theta) \delta_{n}
$$

Therefore,

$$
\begin{aligned}
\left\|w_{n}\right\|^{2}= & \left\langle w_{n}, w_{n}\right\rangle \\
= & \left\langle\theta \delta_{n+1}+(1-\theta) \delta_{n}, \theta \delta_{n+1}+(1-\theta) \delta_{n}\right\rangle \\
= & \theta^{2}\left\|\delta_{n+1}\right\|^{2}+(1-\theta)^{2}\left\|\delta_{n}\right\|^{2} \\
& +2 \theta(1-\theta)\left\langle\delta_{n+1}, \delta_{n}\right\rangle .
\end{aligned}
$$

Note that

$$
-\left(\left\|\delta_{n+1}\right\|^{2}+\left\|\delta_{n}\right\|^{2}\right) \leq 2\left\langle\delta_{n+1}, \delta_{n}\right\rangle \leq\left\|\delta_{n+1}\right\|^{2}+\left\|\delta_{n}\right\|^{2} .
$$

Hence,

$$
\begin{aligned}
& \left\|w_{n}\right\|^{2} \leq \theta\left\|\delta_{n+1}\right\|^{2}+(1-\theta)\left\|\delta_{n}\right\|^{2}, \\
& \left\|w_{n}\right\|^{2} \geq \theta(2 \theta-1)\left\|\delta_{n+1}\right\|^{2}+(1-\theta)(1-2 \theta)\left\|\delta_{n}\right\|^{2} .
\end{aligned}
$$

In view of inequality (39), we have

$$
\begin{aligned}
& \left\|\delta_{n+1}\right\|^{2} \leq\left\|\delta_{n}\right\|^{2}+\left(2 \alpha_{0}+\beta_{0}\right) h\left\|w_{n}\right\|^{2}+\beta_{0} h\left\|w_{n-m}\right\|^{2} \\
& \quad \leq\left\|\delta_{n}\right\|^{2}+\beta_{0} h\left(\theta\left\|\delta_{n+1-m}\right\|^{2}+(1-\theta)\left\|\delta_{n-m}\right\|^{2}\right) \\
& \quad+(2 \theta-1)\left(2 \alpha_{0}+\beta_{0}\right) h\left[\theta\left\|\delta_{n+1}\right\|^{2}-(1-\theta)\left\|\delta_{n}\right\|^{2}\right] .
\end{aligned}
$$

Hence,

$$
\begin{aligned}
& \left\|\delta_{n+1}\right\|^{2} \leq \frac{1+\left(2 \alpha_{0}+\beta_{0}\right) h(1-\theta)(1-2 \theta)}{1-\left(2 \alpha_{0}+\beta_{0}\right) h \theta(2 \theta-1)}\left\|\delta_{n}\right\|^{2} \\
& +\frac{\beta_{0} h}{1-\left(2 \alpha_{0}+\beta_{0}\right) h \theta(2 \theta-1)}\left[\theta\left\|\delta_{n+1-m}\right\|^{2}\right. \\
& \left.+(1-\theta)\left\|\delta_{n-m}\right\|^{2}\right] \\
& \leq \frac{1+\left(2 \alpha_{0}+\beta_{0}\right) h(1-\theta)(1-2 \theta)}{1-\left(2 \alpha_{0}+\beta_{0}\right) h \theta(2 \theta-1)}\left\|\delta_{n}\right\|^{2} \\
& +\frac{\beta_{0} h}{1-\left(2 \alpha_{0}+\beta_{0}\right) h \theta(2 \theta-1)} \\
& \cdot \max \left\{\left\|\delta_{n+1-m}\right\|^{2},\left\|\delta_{n-m}\right\|^{2}\right\} .
\end{aligned}
$$

By induction, we have

$$
\begin{aligned}
& \left\|\delta_{n+1}\right\|^{2} \\
& \quad \leq\left(\frac{1+\left(2 \alpha_{0}+\beta_{0}\right) h(1-\theta)(1-2 \theta)+\beta_{0} h}{1-\left(2 \alpha_{0}+\beta_{0}\right) h \theta(2 \theta-1)}\right)^{k} \\
& \quad \cdot \sup _{-\tau \leq s \leq 0}\|\varphi(s)-\psi(s)\|^{2}, \quad t_{n} \in(k \tau,(k+1) \tau] .
\end{aligned}
$$

The conclusion follows because (42) implies that

$$
\frac{1+\left(2 \alpha_{0}+\beta_{0}\right) h(1-\theta)(1-2 \theta)+\beta_{0} h}{1-\left(2 \alpha_{0}+\beta_{0}\right) h \theta(2 \theta-1)} \in(0,1) .
$$

On the other hand,

$$
x_{n+1}-\bar{x}_{n+1}=\frac{y_{n+1}-z_{n+1}}{\alpha\left(t_{n+1}\right)} .
$$

Therefore, the conclusion holds because $1 / \alpha(t)$ is bounded.

Corollary 21. The implicit Euler method can preserve the asymptotic stability of (1) with $\beta_{0}<-\alpha_{0}$.

Corollary 22. Assume that $1 / \alpha(t)$ is bounded and $\theta \in$ $[1 / 2,1]$. Then the numerical solution (33) is asymptotically stable for (1) satisfying (42). Furthermore, if

$$
1 \geq\left|1+r_{k}\right| \geq\left|1+r_{k-1}\right|, \quad k=0,1,2, \ldots,
$$

the numerical solution (33) is asymptotically stable for (1) satisfying

$$
\beta<\frac{2(2 \theta-1)^{2}}{(2 \theta-1)^{2}+1}(-\alpha) .
$$

It is necessary to find other stability criteria of numerical process (34), since the criteria in Theorem 20 are too tough for numerical scheme (34).

Theorem 23. Assume that $\left|1+r_{k}\right| \leq 1$ and

$$
\alpha+\frac{\beta}{\left|1+r_{k}\right|} \leq 0, \quad k=0,1,2, \ldots
$$

Then the numerical solution $x_{n}$ calculated by numerical process (34) is asymptotically stable for (1) satisfying

$$
\beta \leq-(1-2 \theta)^{2} \alpha .
$$

Proof. Note that (54) implies that $\alpha_{0}+\beta_{0} \leq 0$. Hence, by Lemma 19,

$$
\left\|\delta_{n+1}\right\|^{2} \leq\left\|\delta_{n}\right\|^{2}+h \alpha_{0}\left\|\omega_{n}\right\|^{2}+h \beta_{0}\left\|\omega_{n-m}\right\|^{2},
$$

where

$$
\begin{aligned}
& \alpha_{0}=\alpha, \\
& \beta_{0}=\frac{\beta}{1+r_{k}},
\end{aligned}
$$


By inequality (46), we have

$$
\begin{aligned}
& \left\|\delta_{n+1}\right\|^{2} \leq\left\|\delta_{n}\right\|^{2}+\alpha h\left\|w_{n}\right\|^{2}+\frac{\beta}{\left|1+r_{k}\right|} h\left\|w_{n-m}\right\|^{2} \\
& \leq\left\|\delta_{n}\right\|^{2} \\
& \quad+\frac{\beta}{\left|1+r_{k}\right|} h\left(\theta\left\|\delta_{n+1-m}\right\|^{2}+(1-\theta)\left\|\delta_{n-m}\right\|^{2}\right) \\
& \quad+(2 \theta-1) \alpha h\left[\theta\left\|\delta_{n+1}\right\|^{2}-(1-\theta)\left\|\delta_{n}\right\|^{2}\right] .
\end{aligned}
$$

Therefore,

$$
\begin{aligned}
& \left\|\delta_{n+1}\right\|^{2} \leq \frac{1+\alpha h(1-\theta)(1-2 \theta)}{1-\alpha h \theta(2 \theta-1)}\left\|\delta_{n}\right\|^{2} \\
& +\frac{\left(\beta /\left|1+r_{k}\right|\right) h}{1-\alpha h \theta(2 \theta-1)}\left[\theta\left\|\delta_{n+1-m}\right\|^{2}\right. \\
& \left.+(1-\theta)\left\|\delta_{n-m}\right\|^{2}\right] \leq \frac{1+\alpha h(1-\theta)(1-2 \theta)}{1-\alpha h \theta(2 \theta-1)}\left\|\delta_{n}\right\|^{2} \\
& +\frac{\left(\beta /\left|1+r_{k}\right|\right) h}{1-\alpha h \theta(2 \theta-1)} \max \left\{\left\|\delta_{n+1-m}\right\|^{2},\left\|\delta_{n-m}\right\|^{2}\right\}, \\
& t_{n} \in(k \tau,(k+1) \tau] .
\end{aligned}
$$

Then, by induction, we have

$$
\begin{aligned}
& \left\|\delta_{n+1}\right\|^{2} \leq \prod_{i=0}^{k} \frac{1+\alpha h(1-\theta)(1-2 \theta)+\left(\beta /\left|1+r_{i}\right|\right) h}{1-\alpha h \theta(2 \theta-1)} \\
& \quad \sup _{-\tau \leq s \leq 0}\|\varphi(s)-\psi(s)\|^{2} .
\end{aligned}
$$

Therefore,

$$
\begin{aligned}
& \left\|x_{n+1}-\bar{x}_{n+1}\right\|^{2}=\left\|\frac{y_{n+1}-z_{n+1}}{\alpha\left(t_{n+1}\right)}\right\|^{2} \leq\left(\prod_{i=0}^{k}\left|1+r_{i}\right|\right) \\
& \cdot\left(\prod_{i=0}^{k} \frac{1+\alpha h(1-\theta)(1-2 \theta)+\left(\beta /\left|1+r_{i}\right|\right) h}{1-\alpha h \theta(2 \theta-1)}\right. \\
& \left.\cdot \sup _{-\tau \leq s \leq 0}\|\varphi(s)-\psi(s)\|^{2}\right) \\
& \quad \leq\left[\frac{1+\alpha h(1-\theta)(1-2 \theta)+\beta h}{1-\alpha h \theta(2 \theta-1)}\right]_{-\tau \leq s \leq 0}^{k} \sup _{-} \| \varphi(s) \\
& -\psi(s) \|^{2} .
\end{aligned}
$$

The conclusion follows, since (55) implies that

$$
\frac{1+\alpha h(1-\theta)(1-2 \theta)+\beta h}{1-\alpha h \theta(2 \theta-1)} \in(0,1) \text {. }
$$

Corollary 24. The implicit Euler method can preserve the asymptotic stability of (1) with (54).
Corollary 25. If

$$
r_{k}=0, \quad k=0,1,2, \ldots,
$$

then (54) holds when $\beta<-\alpha$. Then the conclusion in this paper coincides with the numerical stability of delay differential equations in [14].

\section{Numerical Experiments}

In this section, we will do some numerical experiments to illustrate the conclusion.

Consider the following equations:

$$
\begin{aligned}
x^{\prime}(t) & =-x(t)-x^{3}(t)+\frac{1}{3} \sqrt{1+x^{2}(t-1)}, \\
t & \geq 0, t \neq k, \\
\Delta x & =\left(\frac{1}{k+1}-1\right) x\left(k^{-}\right), \quad t=k, k=1, \ldots, \\
x(t) & =t+1, \quad-1 \leq t \leq 0, \\
\bar{x}^{\prime}(t) & =-\bar{x}(t)-\bar{x}^{3}(t)+\frac{1}{3} \sqrt{1+\bar{x}^{2}(t-1)},
\end{aligned}
$$$$
t \geq 0, t \neq k
$$

$$
\begin{aligned}
\Delta \bar{x} & =\left(\frac{1}{k+1}-1\right) \bar{x}\left(k^{-}\right), \quad t=k, k=1, \ldots, \\
\bar{x}(t) & =2, \quad-1 \leq t \leq 0 .
\end{aligned}
$$

We can choose $\alpha(t)=(k+1) !, t \in[k, k+1)$. Then (64) and (65) can be transformed as

$$
\begin{aligned}
& y^{\prime}(t)=-y(t)-\frac{y^{3}(t)}{\alpha^{2}(t)}+\frac{\alpha(t)}{3} \sqrt{1+\frac{y^{2}(t-1)}{\alpha^{2}(t-1)}}, \\
& t \geq 0, t \neq k \text {, } \\
& y(t)=t+1, \quad-1 \leq t \leq 0, \\
& z^{\prime}(t)=-z(t)-\frac{z^{3}(t)}{\alpha^{2}(t)}+\frac{\alpha(t)}{3} \sqrt{1+\frac{z^{2}(t-1)}{\alpha^{2}(t-1)}}, \\
& t \geq 0, t \neq k \text {, } \\
& z(t)=2, \quad-1 \leq t \leq 0 .
\end{aligned}
$$

We chose $h=1 / 10$ and calculated the numerical solutions by numerical process (34) in case of implicit Euler method. Figure 1 shows the relationship of the solutions between (64) and (66). It can be seen from Figure 2 that the difference of solutions to (64) and (65) tends to zero as $n \rightarrow \infty$, which coincides with the conclusion in the paper.

\section{Conflicts of Interest}

The authors declare that they have no conflicts of interest. 

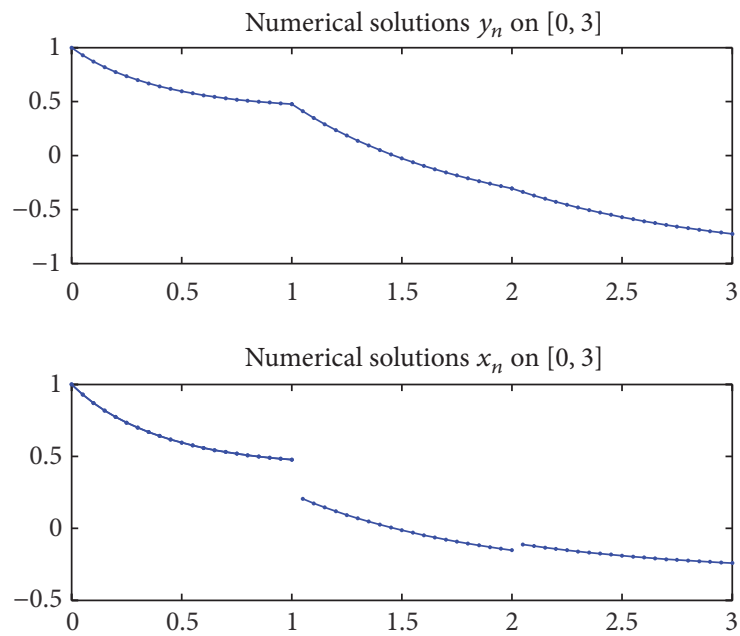

FIGURE 1: Numerical solutions of (64) and $(66)$ on $[0,3]$.

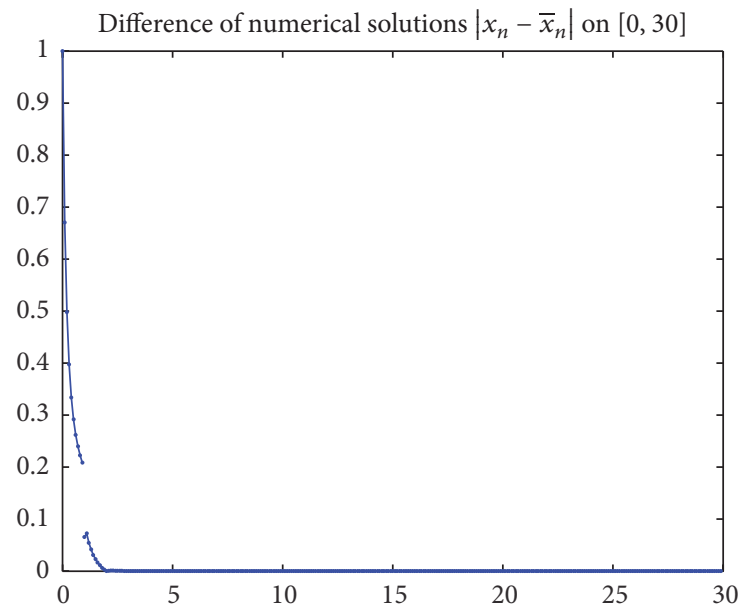

FIGURE 2: The difference of numerical solutions to (64) and (65) on $[0,30]$.

\section{Acknowledgments}

This work is supported by the National Natural Science Foundation of China (Grant no. 11505090) and Shandong Provincial Natural Science Foundation (no. ZR2017BA026 and no. BS2015SF009).

\section{References}

[1] D. D. Bainov and P. S. Simeonov, Systems with Impulsive Effect: Stability, Theory and Applications, Ellis Horwood, Chichester, UK, 1989.

[2] B. Shulgin, L. Stone, and Z. Agur, "Pulse vaccination strategy in the SIR epidemic model," Bulletin of Mathematical Biology, vol. 60, no. 6, pp. 1123-1148, 1998.

[3] L. Stone, B. Shulgin, and Z. Agur, "Theoretical examination of the pulse vaccination policy in the SIR epidemic model," Mathematical and Computer Modelling, vol. 31, no. 4-5, pp. 207-215, 2000.

[4] F. Chen and X. Wen, "Asymptotic stability for impulsive functional differential equation," Journal of Mathematical Analysis and Applications, vol. 336, no. 2, pp. 1149-1160, 2007.
[5] X. Liu and G. Ballinger, "Uniform asymptotic stability of impulsive delay differential equations," Computers \& Mathematics with Applications, vol. 41, no. 7-8, pp. 903-915, 2001.

[6] J. Shen, Z. Luo, and X. Liu, "Impulsive stabilization of functional-differential equations via Liapunov functionals," Journal of Mathematical Analysis and Applications, vol. 240, no. 1, pp. $1-15,1999$.

[7] X. J. Ran, M. Z. Liu, and Q. Y. Zhu, "Numerical methods for impulsive differential equation," Mathematical and Computer Modelling, vol. 48, no. 1-2, pp. 46-55, 2008.

[8] X. Ding, K. Wu, and M. Liu, "The Euler scheme and its convergence for impulsive delay differential equations," Applied Mathematics and Computation, vol. 216, no. 5, pp. 1566-1570, 2010.

[9] G. L. Zhang, M. Song, and M. Z. Liu, "Asymptotical stability of the exact solutions and the numerical solutions for a class of impulsive differential equations," Applied Mathematics and Computation, vol. 258, pp. 12-21, 2015.

[10] G. L. Zhang, M. H. Song, and M. Z. Liu, "Exponential stability of the exact solutions and the numerical solutions for a class of linear impulsive delay differential equations," Journal of Computational and Applied Mathematics, vol. 285, pp. 32-44, 2015.

[11] X. Liu, M. H. Song, and M. Z. Liu, "Linear multistep methods for impulsive differential equations," Discrete Dynamics in Nature and Society, vol. 2012, Article ID 652928, 2012.

[12] J. Yan and A. Zhao, "Oscillation and stability of linear impulsive delay differential equations," Journal of Mathematical Analysis and Applications, vol. 227, no. 1, pp. 187-194, 1998.

[13] X. Liu, G. L. Zhang, and M. Z. Liu, "Analytic and numerical exponential asymptotic stability of nonlinear impulsive differential equations," Applied Numerical Mathematics, vol. 81, pp. 40-49, 2014.

[14] A. Bellen and M. Zennaro, Numerical Methods for Delay Differential Equations, Oxford university press, Oxford, UK, 2003.

[15] S. Li, "High order contractive Runge-Kutta methods for Volterra functional differential equations," SIAM Journal on Numerical Analysis, vol. 47, no. 6, pp. 4290-4325, 2010.

[16] X. Liu and M. Z. Liu, "Asymptotic stability of Runge-Kutta methods for nonlinear differential equations with piecewise continuous arguments," Journal of Computational and Applied Mathematics, vol. 280, pp. 265-274, 2015. 


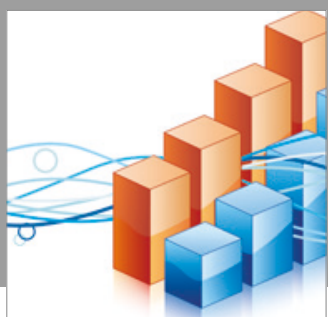

Advances in

Operations Research

vatersals

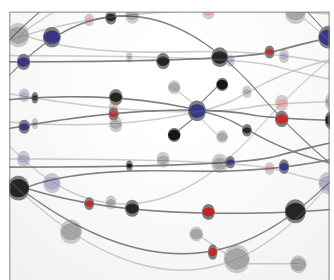

\section{The Scientific} World Journal
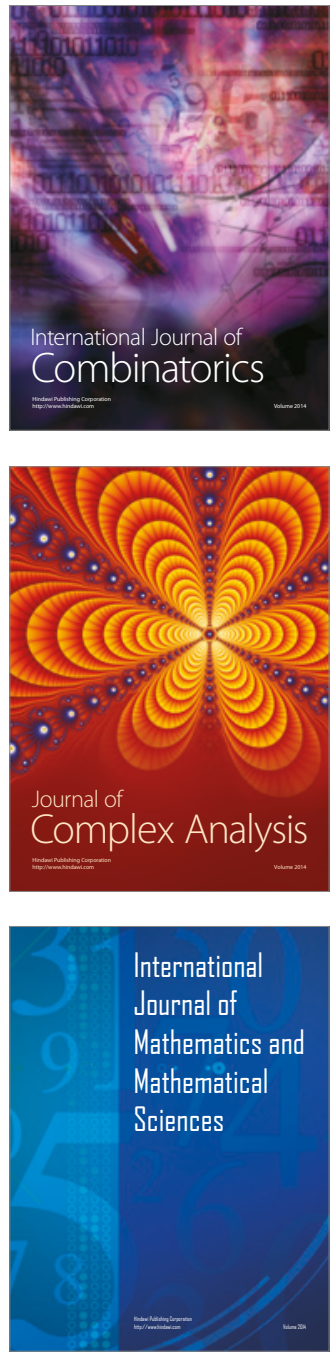
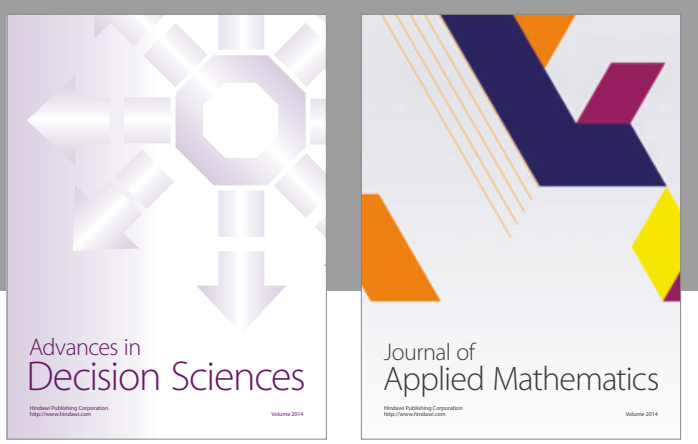

Algebra

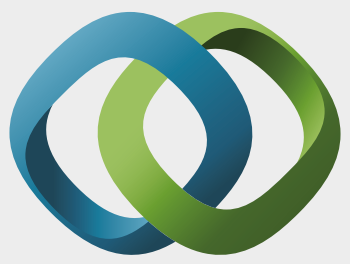

\section{Hindawi}

Submit your manuscripts at

https://www.hindawi.com
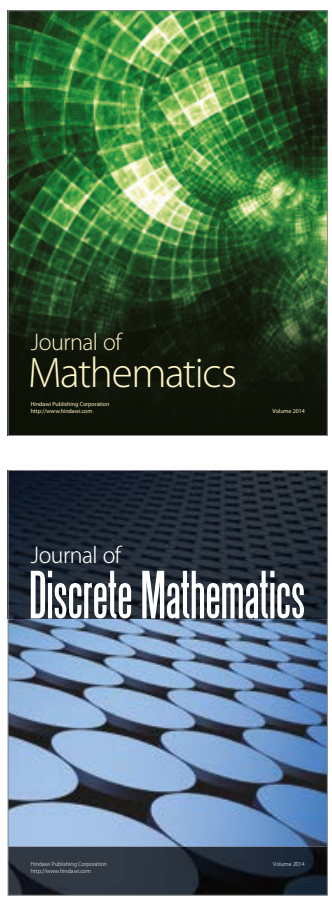

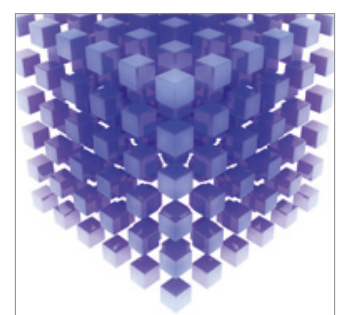

Mathematical Problems in Engineering
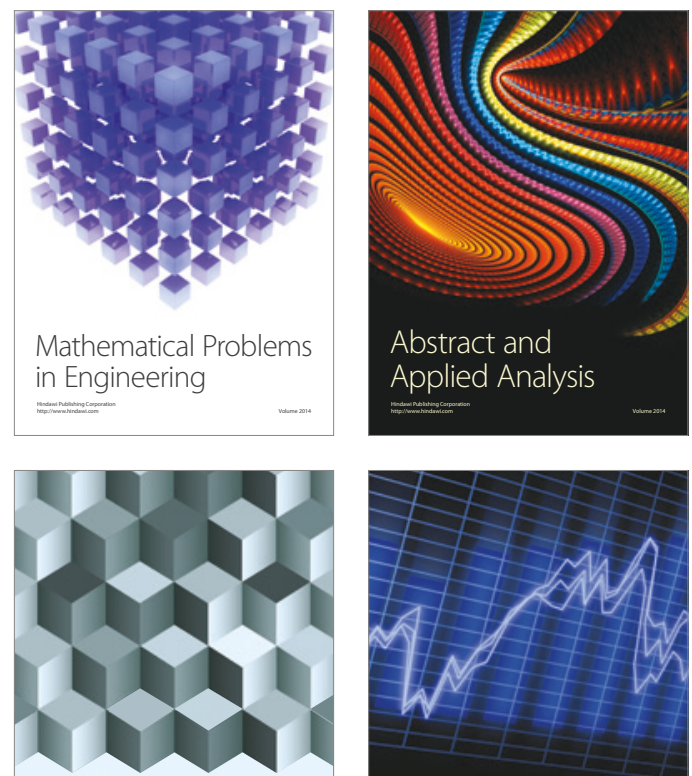

Journal of

Function Spaces

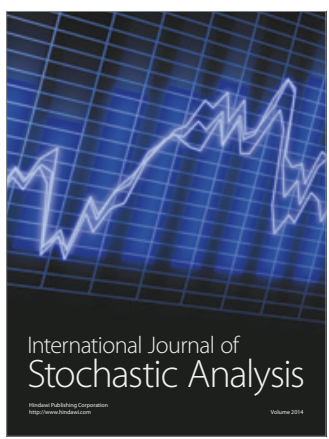

Probability and Statistics
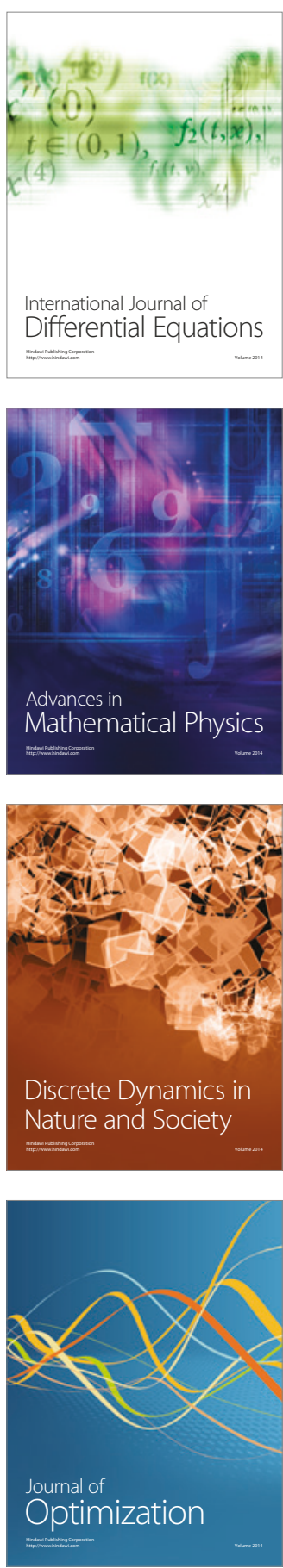